\title{
Observation Result Relationship
} Comment

National Cancer Institute

\section{Source}

National Cancer Institute. Observation Result Relationship Comment. NCI Thesaurus.

Code C70931.

An additional description of the relationship between two or more observation outcomes.

It could refer to the temporal, or sequential relationship, or indicate that a test was

performed to verify another test result, or designate that a test is a component of a test panel. 